\title{
High-resolution computed tomographic characteristics in acute farmer's lung and in its follow-up
}

\author{
Y. Cormier*, M. Brown ${ }^{+}$, S. Worthy ${ }^{+}$, G. Racine*, N.L. Müller ${ }^{+}$
}

\begin{abstract}
High-resolution computed tomographic characteristics in acute farmer's lung and in its follow-up. Y. Cormier, M. Brown, S. Worthy, G. Racine, N.L. Müller. C)ERS Journals Ltd 2000.

ABSTRACT: High resolution computed tomography (HRCT) scans are increasingly used in hypersensitivity pneumonitis (HP). This study looked at HRCT findings at different phases of farmer's lung (FL), a common form of HP.

A cross sectional analysis of 95 HRCT scans of FL cases (20 acute, 75 with a history of FL, 48 still in contact $(E x+)$ (dairy farm), and 27 who had ceased contact (Ex-)) was made. All scans were read independently by two, and if needed by three, radiologists blinded to the category. The lungs were divided into six regions (fives lobes + lingula), and read for attenuation/mosaic, ground-glass, micronodules, fibrosis, and emphysema. A score of $0-3$ was given for each region and each variable: $0=$ absence, 1 $=<\mathbf{2 5 \%}$ of the surface, $2=\mathbf{2 5}-\mathbf{5 0} \%, 3=>\mathbf{5 0} \%$. Mediastinal lymphadenopathy was also noted.
\end{abstract}

Ground glass, predominating in the lower lobes, was the most frequent feature in the acute and $\mathbf{E x}+$ cases. Other abnormalities had no preferential distribution. $\mathbf{E x}+$ had more ground-glass than the $\mathbf{E x}-(\mathrm{p}=\mathbf{0 . 0 0 2 5})$. Emphysema was more frequently seen than interstitial fibrosis $(p=0.004)$. Mediastinal lymphadenopathy was present in 26 cases (9 acute, $10 \mathrm{Ex}+$ and $7 \mathrm{Ex}-$ ).

In conclusion, in farmer's lung: 1) ground-glass predominates in the lower lobes while the other abnormalities have no anatomic predilection; 2) contact avoidance allows a better resolution of computed tomography abnormalities than continued exposure; 3) emphysema is a more frequent finding than interstitial fibrosis; and 4) the presence of mediastinal lymphadenopathy has no negative diagnostic value. Eur Respir J 2000; 16: 56-60. \author{
VHHSC, Vancouver BC, Canada. \\ Correspondence: Y. Cormier \\ Hôpital Laval \\ 2725 Chemin Ste Foy \\ Ste Foy \\ P.Q. Canada \\ G1V 4G5 \\ Fax: 14186564762
}

*Unité de Recherche, Centre de Pneumologie, Hôpital and Université Laval, Ste Foy, Quebec and ${ }^{+}$Dept of Radiology,

Keywords: Emphysema

hypersensitivity pneumonitis

interstitial lung disease

Received: July 71999

Accepted after revision March 92000
Hypersensitivity pneumonitis (HP) is an interstitial lung disease caused by an immune response to a variety of antigens [1]. The sources of antigens vary between different regions of the world; in Canada the most frequent types are farmer's lung, caused by a thermophilic bacteria (Saccharopolyspora rectivirgula), which grows in mouldy hay, bird fancier's disease (animal proteins), and humidifier's lung (Penicillium sp.).

The diagnosis of this disease is based on a variety of nonspecific and nonvalidated criteria [1-4]. The use of chest radiographs has always been an important diagnostic parameter [5]. More recently, high resolution computed tomography (HRCT) has become a very useful tool in the diagnosis of HP. The findings of HRCT in HP have been described [6-10]. In both acute and chronic HP, HRCT can help distinguish this disease from other interstitial lung diseases $[6,11]$. Being more sensitive than standard chest radiographs, HRCT will identify abnormalities where none were seen on posterioanterior and lateral chest films [12]. Although reported as normal in few cases [13], the absence of abnormalities on HRCT is a strong argument against the diagnosis of active HP. More importantly, HRCT will yield a much more precise description of the underlying abnormalities allowing better understanding of the disease process. In active disease typical HRCT shows diffuse micronodules and patchy ground-glass attenuation $[6,7]$. These typical findings are very useful in the differential diagnosis of HP from other causes of interstitial diseases; in one study computed tomography (CT) allowed correct distinction of HP from idiopathic pulmonary fibrosis in $90 \%$ of cases [9]. In more chronic or residual disease the findings include focal air trapping or emphysema and mild fibrosis $[7,14,15]$. The fibrosis is typically the middle lung zones and this pattern can help distinguish HP sequella from other causes of lung fibrosis [7]. HRCT can identify emphysematous changes in patients with a history of farmer's lung, changes often not detected on standard chest radiographs [15]. Previous studies have usually reported only a small number of cases or, more importantly, have not thoroughly addressed the findings in patients with a past history of this disease. The present authors and others have previously shown that emphysema is an important sequela of farmer's lung $[14,16]$. In pigeon breeder's disease, interstitial fibrosis was the most important outcome in one study [17] while in another report $44 \%$ of chronic cases had emphysema [15].

The purpose of this study was to look at and describe the findings on HRCT in a large group of patients with farmer's lung both for the acute phase and during the long 
term outcome after the acute episode. Of particular interest was verifying if HRCT could identify an effect of continued exposure versus contact avoidance in the outcome of these patients.

\section{Materials and methods}

\section{Study population}

Ninety five HRCT scans were obtained from 89 patients at diagnosis of active farmer's lung $(n=20)$, during clinical remission during follow-up between 2 months and 25 yrs after the acute event (Mean of $7.7 \mathrm{yrs}, \mathrm{n}=75$ ). Of these 75, 48 still worked on the farm while 27 had not been exposed to the environment for at least 2 years. There were 20 exsmokers and one current-smoker. The mean pack years of cigarette consumption was 27 and the mean time since quitting smoking was 19 yrs. The diagnosis of HP was based on previously published criteria [1-4]. It was based on a combination of the following parameters appropriate exposure (dairy barn), compatible symptoms (recurrent febrile episodes, dyspnoea), inspiratory crackles on physical examination, restrictive lung disease with decreased lung diffusion capacity, interstitial markings on chest radiographs and/or HRCT, BAL lymphocytosis, granulomas on transbronchial biopsies, serum precipitating antibodies to common farmer's lung antigens, and rarely, surgical lung biopsy.

\section{High resolution computed tomography}

All scans were obtained with a Toshiba TCT-900S. Nine 1 -mm collimation scans, spaced $1.5 \mathrm{~cm}$ apart, were obtained for each subject. All CT scans were interpreted separately by two independent observers. In cases where there were discrepancies between the two readers, a third radiologist was consulted and a final score was attributed either by consensus or by a majority of $2: 1$. Scoring was done for each lobe and the lingula separately (six regions per subject). A gradation of 0-3 was given for each anatomical region for the following descriptors: 1) decreased attenuation/mosaic perfusion; 2) ground-glass; 3) micronodules; 4) reticular pattern/honeycombing (fibrosis; and 5) emphysema.

The HRCT findings were defined according to the recommendations of the nomenclature committee of the Fleischner Society [18]. Mosaic perfusion was considered present when there was a patchwork of regions of different attenuations, interpreted as being secondary to regional differences in perfusion. This interpretation was based on the presence of increased size and number of vessels in areas with increased attenuation compared to areas of decreased attenuation. In this setting the areas of decreased attenuation are presumed to be due to air trapping. Ground-glass attenuation was defined as the presence of hazy increased opacity of the lung without obscuration of the underlying bronchial and vascular margins. The distinction of increased attenuation resulting from mosaic perfusion from ground-glass attenuation resulting from interstitial and airspace disease is based on the number and size of vessels compared to the remaining lung. In areas of increased attenuation resulting from mosaic perfusion the number and size of vessels is increased compared with the remaining lung whereas in areas of ground-glass attenuation resulting from interstitial or airspace disease the number and size of vessels is similar to that of the remaining lung. Micronodules were defined as discrete, small, round, focal opacities measuring no more than $7 \mathrm{~mm}$ in diameter. A reticular pattern was defined as the presence of innumerable, interlacing linear opacities resulting in an appearance suggesting a mesh. Honeycombing was considered present when HRCT demonstrated clustered cystic air spaces with welldefined walls and measuring $0.3-1.0 \mathrm{~cm}$ in diameter. Emphysema was considered present when HRCT demonstrated focal regions of low attenuation without visible walls.

Emphysema was defined by the presence of focal regions of low attenuation, usually without visible walls, resulting from actual or perceived enlarged air spaces and destroyed alveolar walls. Mosaic attenuation was defined as the presence of a patchwork of regions of varied attenuation. These definitions are from the recommendations of the Nomenclature committee of the Fleischner Society [18]. Focal areas of attenuation near the centre of the secondary lobule were interpreted as being due to centrilobular emphysema. Localized areas of attenuation, which had a lobular or segmental pattern, were interpreted as presumably being due to small airway disease.

For each descriptor, a score of 0 represents no anomaly, 1 involvement of $<25 \%$ of the lobe, 2 involvement of $25-$ $50 \%$, and $3>50 \%$. The presence of any adenopathy with a shorter axis $>10 \mathrm{~mm}$ was noted and the American Thoracic Society classification station for each enlarged node identified.

\section{Statistical analysis}

For graphical representation of the data, results of representative measures were expressed as mean \pm SEM. Two approaches were performed to analyse data from the sum of each radiological descriptor. The first one being, for each descriptors, the comparison between Acute, $\mathrm{Ex}+$ and Ex-. The statistical method used to perform this analysis was a one-way ANOVA with a factor representing the group effect (the comparison between the three groups). Normality and variance assumptions were tested. Posteriori comparisons were performed with Tukey's method. Results of statistical differences are expressed with letters in minuscule above each column.

The second analysis was to compare the descriptors for each group. Data were analysed using a randomized block design. Also, normality and variance assumptions were tested and the posteriori comparisons were performed with Tukey's method.

A correspondence analysis (CA) was done on each group of subjects to describe the relationship among the five descriptors for the three anatomical regions of the right lung (upper, middle, and lower lung) and the grade levels $(0-3)$. The results of these analyses are presented in figure 2. These graphs represent the configuration of points in the best projection plane formed by the first two principal axes. The portion of variance gives a conservative idea of the percentage of information accounted for by the principal axes. The interpretation of results can be made from 


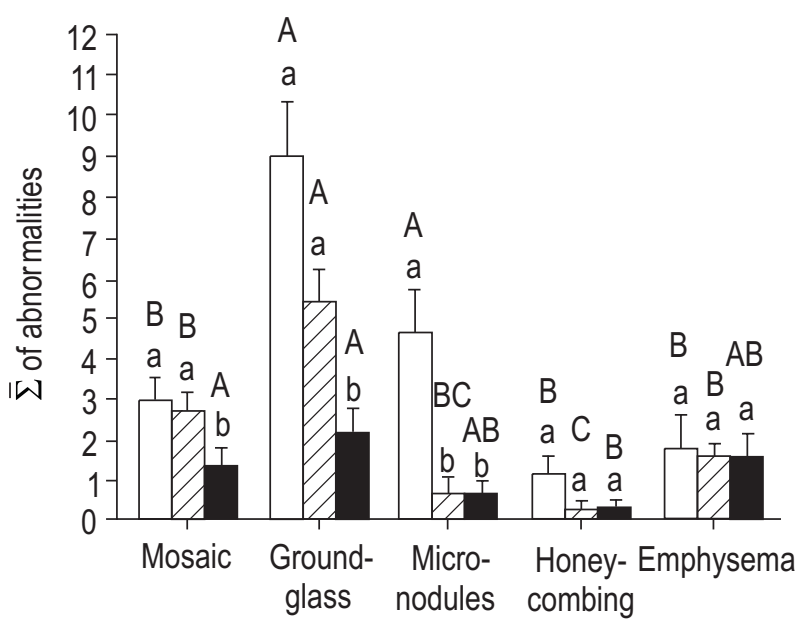

Fig. 1. - Mean \pm SEM of the individual mean of the sum $(\bar{\Sigma})$ of the intensity score for each of the five lung field descriptors evaluated for three groups of subjects: acute farmer's lung $(\square)$, farmers with a history of farmer's lung still in contact with the environment $\left(\mathrm{Ex}^{+}, \mathbb{Z}\right)$ and those who were no longer exposed to the farm $(\mathrm{Ex}-, \boldsymbol{\square})$. The lungs were divided into six regions and each region was given a score of $0-3$ for each of the descriptors, therefore the maximal possible score was 18 . Lower case letters above each column compare values for a given descriptor for the three groups, whilst uppercase letters compare results between different descriptors within each group of subjects; columns with different letters are significantly different $(\mathrm{p}<0.05)$. For example, there was significantly more ground glass in the acute than the Ex-, the difference between the acute and the Ex + did not reach statistical significance. Ground-glass was the most prevalent abnormality in the acute and the Ex+ but not for the Ex-.

the proximities (using chi-square distance as the metric) among descriptors, among grade levels, and finally among descriptors and grade levels. Two descriptors whose corresponding points are close to one another have similar profiles for gradation, i.e. similar distributions. In the same manner, two grade levels whose corresponding points are close to one another have similar profiles for the lung descriptors. It is also legitimate to interpret the relative position of one descriptor (or one grade level) with respect to all the grade levels (or to all the descriptors). For example, a descriptor has a profile characterized by a high percentage on the grade levels whose corresponding points are nearby the descriptor. The origin of the axes corresponds to the average profiles of both descriptors and grade levels. Thus a descriptor near the origin has an undifferentiated distribution among the grade levels. It has a distribution similar to the one obtained with the average of all descriptors.

Fisher's exact test was used to measure the relation between smoking and emphysema. The data were analysed using the statistical package program SAS (SAS Institute Inc., Cary, NC, USA).

\section{Results}

The data summarizing the lung involvement, as evaluated by the CT scans, are presented in figure 1 . This figure represents the mean \pm SEM values of the sum of each radiological descriptor for the three groups of subjects. Ground glass was by far the most predominant finding in acute cases and in the Ex+ subjects. Ex-group still had some ground-glass, but this pattern was not more prevalent than other abnormalities except fibrosis and less prevalent than in either of the two other groups. For all subjects there was more emphysema than honeycombing/fibrosis $(\mathrm{p}=$ $0.004)$. Within groups this was significant only for the $\mathrm{Ex}+$. There was radiological evidence of emphysema in 31 of the 95 scans evaluated; of these, 13 were exsmokers, one was a current-smoker and 17 had never smoked. Seven subjects with emphysema also had radiological evidence of interstitial fibrosis. As shown in table 1 as expected smoking was a risk factor for emphysema. A large number of subjects with emphysema however, had never smoked. The length of time since the initial diagnosis of the HP event was not related to any of the five lung parameters tested: attenuation/mosaic $\mathrm{R}=0.006, \mathrm{p}=0.96$, ground-glass $\mathrm{R}=0.07, \mathrm{p}=0.6$, micronodules $\mathrm{R}=0.009, \mathrm{p}=$ 0.94 , fibrosis $\mathrm{R}=0.93, \mathrm{p}=0.45$, and emphysema $\mathrm{R}=0.05$, $\mathrm{p}=0.66$.

Six subjects had two HRCT scans, four cases changed categories between the two evaluations (i.e. from acute to $\mathrm{Ex}+$ or Ex-). Two subjects in the $\mathrm{Ex}+$ had two HRCT while in this category. In one case, where the two HRCT were two years apart, the scores were identical, In the other case, 5 yrs separated the two evaluations, the second HRCT had a lower score for ground-glass (total ground-glass scores 15 and 9 respectively).

A graphical representation of the correspondence analysis of the radiological data for the right lungs is presented in figure 2. This presentation shows that the ground-glass images were progressively more important as one moved down from the upper to the middle and lower lobes, while all other abnormalities had no specific anatomic predilection. Distributions for the left lungs were similar to those of the right lungs (data not presented).

Abnormal mediastinal adenopathy, were seen on 26 HRCT scans of 25 patients: nine acute, $10 \mathrm{Ex}+$ and seven Ex- (fig. 3). Nodes most often involved were the right lower paratracheal (No. 4R), the subaortic (No. 5), subcarinal (No. 7) and the right hilar (No. 10R) [19]. As described in the definition, all lymph nodes deported had a shorter diameter of $10 \mathrm{~mm}$. No nodes $>20 \mathrm{~mm}$ were seen.

\section{Discussion}

This study describes the HRCT findings in a large group of patients with current or past history of farmer's lung. The importance of ground-glass radiological abnormality in this disease is confirmed [6,7]. It is unclear if this geographical distribution represents more disease activity in the lower lung regions or a gravitational effect. Although all scans were performed with the subjects in the supine position, the top-base pressure gradient would still favour less expansion of the lower lobes, perhaps explaining some of this finding. The fact that sequelae of the disease, both in terms of emphysema and lung fibrosis, do not predominate in the lower lobes, support the hypothesis that there is not more disease in these lower lung regions. More fibrosis in the middle lung fields, as previously reported, was not found [7].

The observation that farmers with a history of farmer's lung who remain on the farm, had more ground-glass abnormalities than those who had quit the farm, although they had no symptoms of ongoing disease, suggests that the previously described persistent lymphocytic alveolitis 
a)

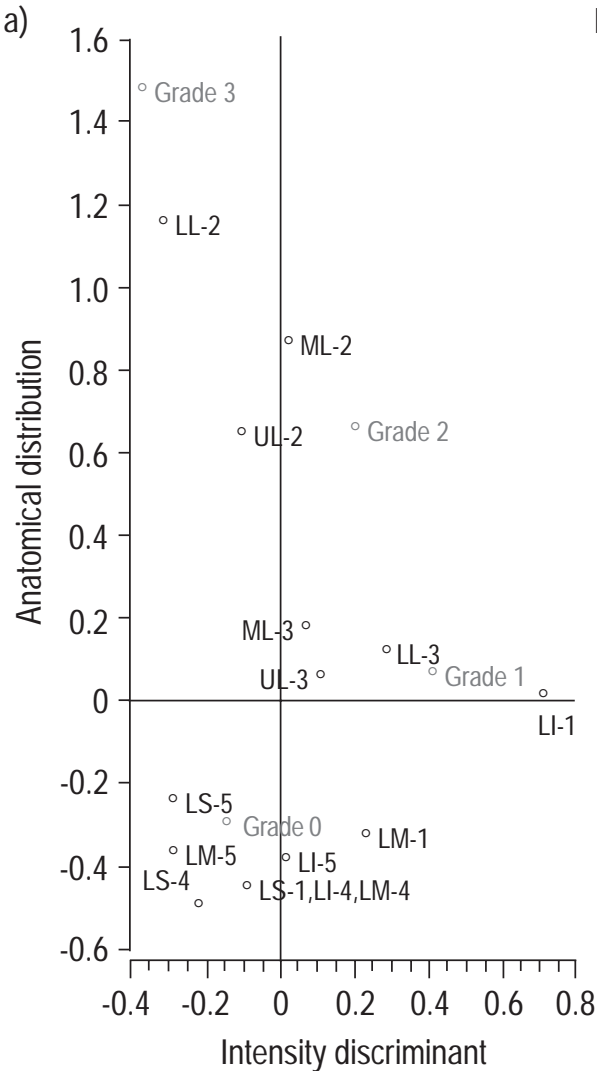

b)

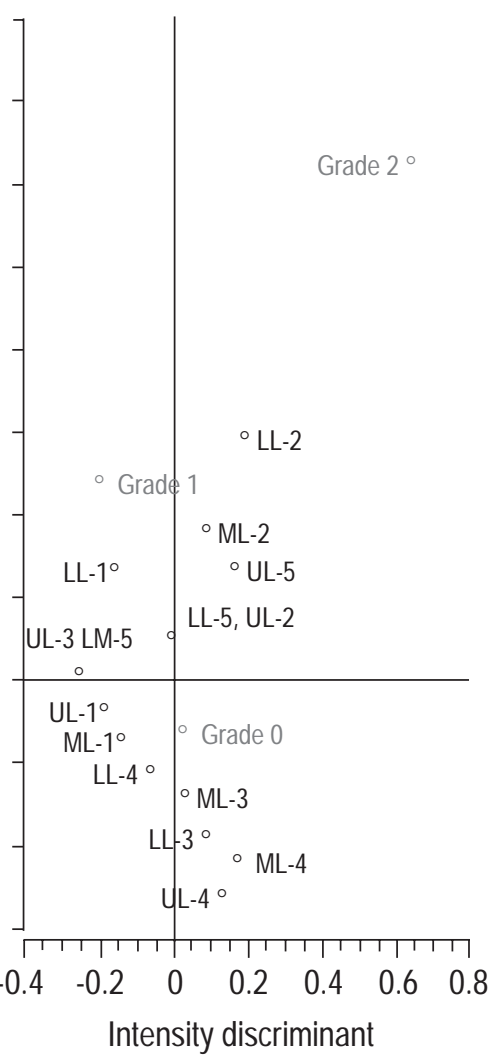

c)

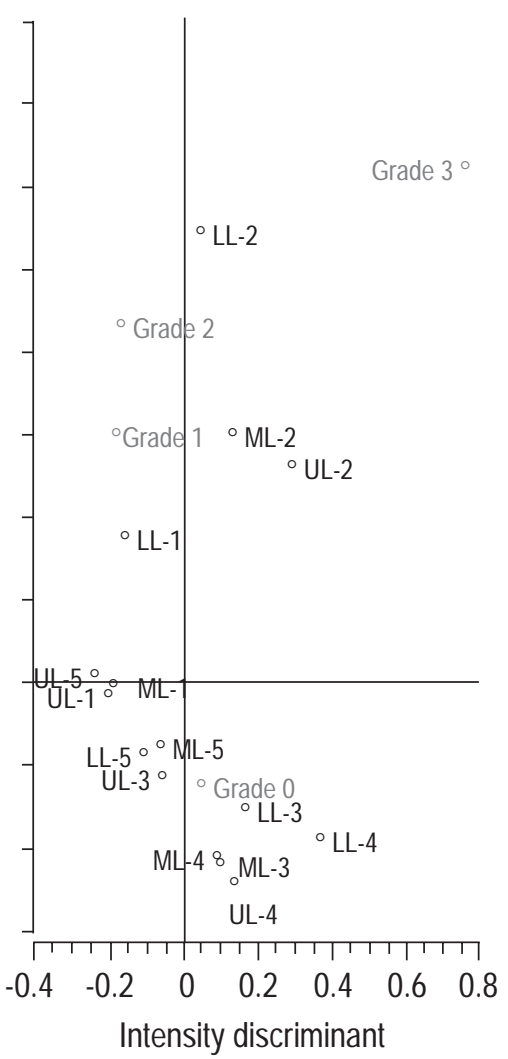

Fig. 2. - Correspondence analysis of the radiological data for the right lungs of: a) subjects with acute farmer's lung; b) farmers with a history of farmer's lung and still in contact with the environment (Ext); and c) farmers with a history of farmer's lung but no longer exposed to the farm groups. Note, as seen in figure 1, the predominance of ground-glass in acute and Ex+ groups. The vertical axis, which is the predominant component of the graph, shows the anatomical distribution of the five descriptors. Note that the ground-glass abnormalities are progressively more pronounced from the upper (UL) through the middle (ML) and lower (LL) lobes. This anatomical predilection is not apparent for the other descriptors. Types of abnormalities are: 1 : mosaic; 2 : ground-glass; 3: micronodules; 4: honeycombing; and 5: emphysema. The proportion of variance explained by the x-axes are $18.39,19.12$ and $9.9 \%$ for $\mathrm{a}, \mathrm{b}$ and $\mathrm{c}$ respectively; and $68.24,80.88$ and $86.51 \%$ for $\mathrm{a}, \mathrm{b}$ and $\mathrm{c}$ respectively for the y-axes.

[20] in these subjects may represent an ongoing disease process and not a normal immune response as previously believed. The ground-glass abnormalities could however only reflect the lymphocytic alveolitis and not represent ongoing disease per se. Prospective long term follow-up studies are needed to verify if these abnormalities translate in more permanent lung damage over time.

The results of this study confirm previous reports that emphysema is a more prevalent long term outcome of farmer's lung than interstitial lung fibrosis $[14,16]$. This is different than what has been reported in pigeon breeder's disease, where lung fibrosis is the major outcome [17].

Table 1. - Relationship between smoking and emphysema

\begin{tabular}{lrcr}
\hline & \multicolumn{3}{c}{ Emphysema } \\
\cline { 2 - 4 } & No & Yes & Total \\
\hline Smoking history & 9 & 13 & 22 \\
Ex & 54 & 17 & 71 \\
None & 1 & 1 & 2 \\
Current & 64 & 31 & 95 \\
Total &
\end{tabular}

Note that although smoking was associated with the presence of emphysema ( $p=0.009$, Fisher's exact test) the majority of subjects with emphysema had never smoked.
Although emphysema was more prevalent in exsmokers than in nonsmokers, the fact that $60 \%$ of exsmokers and one out of two current-smokers with a mean smoking history of 27 pack years and $24 \%$ of never-smokers had emphysema strongly suggest that the emphysema observed in our subjects is a direct consequence of HP. The emphysema seen in farmers with farmer's lung also cannot be explained by farming alone [16].

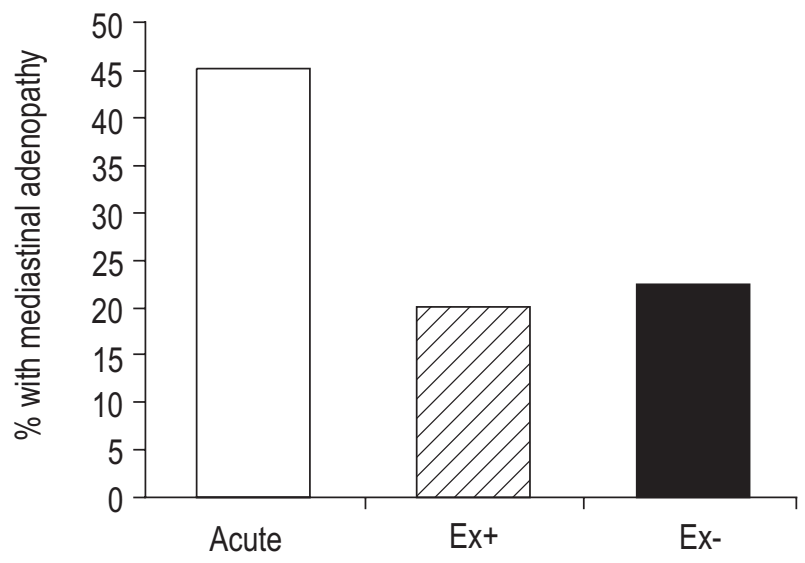

Fig. 3. - Percentage of subjects from the three groups studied with enlarged mediastinal lymph nodes (smaller diameter $>1 \mathrm{~cm}$ ). 
The presence of enlarged lymph nodes in HP was previously described for acute and subacute HP [21]. In this study the presence of enlarged lymph nodes in subjects with a history of HP with no current clinically active disease suggests that the presence of mediastinal adenopathy may not be a reliable finding to rule out HP or a history of the disease or differentiate it from sarcoidosis or other clinical entities associated with enlarged mediastinal lymph nodes.

In conclusion, this study showed that in farmer's lung ground-glass predominates in the lower lobes, while the other abnormalities have no anatomic predilection; contact avoidance allows a better resolution of computed tomography abnormalities than continued exposure; emphysema is a more frequent finding than interstitial fibrosis; and the presence of mediastinal lymphadenopathy has no negative diagnostic value.

\section{References}

1. Bone RC, ed. Part M; interstitial lung disease. In: Cormier Y, Schuyler M, eds. Hypersensitivity Pneumonitis. Textbook of Pulmonary Medicine. St Louis, MO, Mosby-Year Book, 1992; pp. 1-9.

2. Chemlik F, Dolico G, Reed CE, Dickie H. Farmer's lung. J Allergy Clin Immunol 1975; 54: 180-188.

3. Richerson HB, Bernstein LL, Fink HN, et al. Guidelines for the clinical evaluation of hypersensitivity pneumonitis. J Allergy Clin Immunol 1989; 84: 839-844.

4. Terho EO. Diagnostic criteria for farmer's lung disease. Am J Ind Med 1986; 10: 329.

5. Hodgson MJ, Parkinson DK, Karpf M. Chest X-Rays in hypersensitivity pneumonitis: a meta-analysis of secular trend. Am J Indust Med 1989; 16: 45-53.

6. Silver SF, Müller NL, Miller RR, Lefcoe MS. Hypersensitivity pneumonitis: evaluation with CT. Radiology 1989; 173: 441-445.

7. Adler BD, Padley SP, Muller NL, Remy-Jardin M, Remy J. Chronic hypersensitivity pneumonitis: high resolution CT and radiographic features in patients. Radiology 1992; 185: 91-95.

8. Buschman DL, Gamsu G, Waldron JA Jr, Klein JS, King TE Jr. Chronic hypersensitivity pneumonitis: use of CT in diagnosis. Am J Roentgenol 1992; 159: 957-960.

9. Lynch DA, Newell JD, Logan PM, King TE, Muller NL. Can CT distinguish hypersensitivity pneumonitis from idiopathic pulmonary fibrosis? Am J Roentgenol 1995; 165: $807-811$
10. Vincent JM, Flower CD, Shneerson JM, Stewart S. Extrinsic allergic alveolitis: problems in diagnosis and a potential use for computed tomography. Respir Med 1992; 86: 135-141.

11. Nishimura K, Izumi T, Kitaichi M, Nagai S, Itoh H. The diagnostic accuracy of high-resolution computed tomography in diffuse infiltrative lung diseases. Chest 1993; 104: 1149-1155.

12. Akira M, Kita N, Higashihara T, Sakatani M, Kozuka T. Summer-type hypersensitivity pneumonitis: comparison of high-resolution CT and plain radiographic findings. $\mathrm{Am}$ J Reontgenol 1992; 158: 1223-1228.

13. Lynch DA, Rose CS, Way D, King TE Jr. Hypersensitivity pneumonitis: sensitivity of high-resolution CT in a population-based study. Am J Roentgenol 1992; 159: 469-472.

14. Lalancette M, Carrier G, Ferland S, et al. Long term outcome and predictive value of bronchoalveolar lavage fibrosing factors in farmer's lung. Am Rev Respir Dis 1993; 148: 216-221.

15. Remy-Jardin M, Remy J, Wallaert B, Muller N. Subacute and chronic bird breeders hypersensitivity pneumonitis: sequential evaluation with $\mathrm{CT}$ and correlation with lung function tests and bronchoalveolar lavage. Radiology 1993; 189: 111-118.

16. Erkinjuntti-Pekknen R, Rytkönen H, Kokkarinen JI, Tukiainen HO, Partanen K, Terho EO. Long-term risk of emphysema in patients with farmer's lung and matched control farmers. Am J Respir Crit Care Med 1998; 158: 662-665.

17. Pérez-Padilla R, Salas J, Chapela R, et al. Mortality in Mexican patients with chronic pigeon breeder's lung compared to those with usual interstitial pneumonitis. Am Rev Respir Dis 1993; 148: 49-53.

18. Austin JHM, Muller NL, Freedman PJ, et al. Glossary of terms for CT of the lungs: recommendations of the Nomenclature committee of the Fleischner Society. Radiology 1996; 200: 327-331.

19. American Thoracic Society. Clinical staging of primary lung cancer. Am Rev Respir Dis 1983; 126: 1-6.

20. Leblanc P, Bélanger J, Laviolette M, Cormier Y. Farmer's lung disease: Relationship between continued exposure, alveolitis, and clinical status. Arch Int Med 1986; 146: 153-157.

21. Niimi H, Kang KY, Kwong S, Carignan S, Muller NL. CT of chronic infiltrative lung disease: prevalence of mediastinal lymphadenopathy. J Comp Assist Tomogr 1996; 20: 305-308. 